\title{
Task Force Tarmed
}

Die Gesamtrevision des Tarmed-Tarifs ist vor wenigen Tagen mit dem Ende der Vernehmlassung durch die Ärzteschaft in eine neue Phase getreten. Mehrere tausend Feedbacks, darunter einige Hundert betreffend die bildgebenden Verfahren, müssen nun gesichtet, klassiert und je nach Relevanz in den Entwurf des neuen Tarifwerks eingebaut werden.

Sehr große Resonanz, teilweise sogar Empörung, hat die frühere Mitteilung über die geplante Streichung der qualitativen Dignität aus dem zukünftigen Tarifwerk ausgelöst. Inzwischen wurde deshalb doch eine reduzierte Zuordnung von Positionen $\mathrm{zu}$ entsprechend qualifizierten Leistungserbringern zugelassen. Dies führte erwartungsgemäß zu nicht geringen „Feuerwehr-Übungen“, gehört doch gerade auch der Ultraschall in die Hände von gut ausgebildeten Ärzten. Es bleibt zu hoffen, dass diese großen Anstrengungen denn auch fruchtbar bleiben.

Nun gilt es für den Tarifentwurf, einige gewichtige Hürden zu nehmen. Interessant ist vor allem die zeitliche Abfolge, besteht doch die Befürchtung, dass nach erfolgter Absegnung durch die Ärzteschaft noch Kostenträger und politische Instanzen relevante Änderungen am Tarifwerk vornehmen werden. Dass diese wohl kaum zugunsten der Leistungserbringer ausfallen werden, muss jetzt schon mit einer gewissen Resignation erwartet werden. 